\title{
Editorial
}

\section{Advances in Coastal HF and Microwave (S- or X-Band) Radars}

\author{
Weimin Huang, ${ }^{1}$ Xiongbin $\mathrm{Wu}^{2}{ }^{2}$ Björn Lund, ${ }^{3}$ and Khalid El-Darymli ${ }^{4}$ \\ ${ }^{1}$ Faculty of Engineering and Applied Science, Memorial University, St. John's, NL, Canada A1B 3X5 \\ ${ }^{2}$ School of Electronic Information, Wuhan University, Wuhan, Hubei 430072, China \\ ${ }^{3}$ Rosenstiel School of Marine and Atmospheric Science, University of Miami, Miami, FL 33149, USA \\ ${ }^{4}$ Northern Radar Inc., St. John's, NL, Canada A1B 3E4 \\ Correspondence should be addressed to Weimin Huang; weimin@mun.ca
}

Received 24 October 2016; Accepted 24 October 2016; Published 28 February 2017

Copyright (C) 2017 Weimin Huang et al. This is an open access article distributed under the Creative Commons Attribution License, which permits unrestricted use, distribution, and reproduction in any medium, provided the original work is properly cited.

Ground-based high frequency $(3-30 \mathrm{MHz})$ surface wave radar (HFSWR) and microwave (S-band: $\sim 3 \mathrm{GHz}$ or X-band: $\sim 10 \mathrm{GHz}$ ) marine radar have been widely used in ocean remote sensing for over four decades. HFSWR systems are able to monitor sea surface currents, winds, waves, and targets "over the horizon" (up to $300 \mathrm{~km}$ ) due to the strong interaction between HF radio signals and ocean gravity waves. Compact microwave marine radar covers the "line-of-sight" area with a relatively high spatial resolution finer than $10 \mathrm{~m}$ and can be deployed on ships or land. This enables S-band or $\mathrm{X}$-band radar to be an ideal sensor for marine navigation or provide finer sea surface currents, waves, and winds measurement for the area of interest.

Due to increasing development of HF radar networks worldwide and new applications of regular microwave marine radars for expanding coastal ocean observing capabilities and improving safety of human marine activity, the purpose of this special issue is to present the state-of-the-art developments and associated challenges in ocean remote sensing applications by leading researchers and practitioners of both $\mathrm{HF}$ and microwave radars.

Eight papers related to HF radar research are included in this issue. Both the first- and second-order bistatic radar ocean cross sections for an antenna on a floating platform have been derived for the case of a frequency-modulated continuous-wave (FMCW) source in the paper titled "Bistatic High Frequency Radar Ocean Surface Cross Section for an FMCW Source with an Antenna on a Floating Platform." The effect of platform motion on the radar cross sections for an FMCW waveform is investigated and compared with that for a pulsed source based on simulated data. The results have important implications in the application of increasing bistatic HFSWR for ocean remote sensing and will possibly improve corresponding performance. In the paper titled "Observation of Zenneck-Like Waves over a Metasurface Designed for Launching HF Radar Surface Wave," an infrared method is used to visualize a Zenneck-like wave above a metasurface located on a dielectric slab based on simulation and experimentation. The work may provide a way to enhance surface wave excitation for HFSWR. A general range-velocity processing scheme for the discontinuous spectrum-FMCW (DS-FMCW) HF radar applications is presented in the paper titled "A General Range-Velocity Processing Scheme for Discontinuous Spectrum FMCW Signal in HFSWR Applications." The effectiveness of the proposed scheme is validated based on satisfactory target detection results from simulated data. Ionospheric clutter contamination has been a problem that affects the performance of HFSWR for a long time. Based on field data, the characteristics of the first-order sea echoes and ionospheric interferences are analyzed in the paper titled "Cross Spectral Analysis of CODAR-SeaSonde Echoes from Sea Surface and Ionosphere at Taiwan." The results show that the sea and ionospheric echoes in the CODAR-SeaSonde observed cross power spectrum may be distinguished according to the discrepancies in the characteristics of the coherence and phases between the sea and ionospheric echoes. In the paper titled "The Vertical Ionosphere Parameters Inversion for High Frequency Surface Wave Radar," an inversion method utilizing range-Doppler spectrum of HFSWR radar is proposed to extract ionosphere information, such as the electron density, the plasma frequency of irregularities, and the plasma drift velocity 
of irregularities, from the ionospheric clutter. An artificial neural network (ANN) based method to estimate the wind speed from HF radar data is presented in the paper titled "Wind Speed Inversion in High Frequency Radar Based on Neural Network." The method utilizes the ANN to map the relationship between the wind speed and the sea state parameters. The results from a one-month-long $13 \mathrm{MHz}$ SeaSonde HFSWR's data collected in 2012 showed that the ANN is an efficient tool for wind speed estimation from HFSWR data in operational scenarios. The paper titled "A New Method of Wave Mapping with HF Radar" retrieves the significant wave height from the ratio of the second-harmonic peak to the Bragg peak. This method can be applied to both narrow and broad-beam HF radars, and results are in good agreement with reference buoy data. The review paper titled "Application of HF Radar in Hazard Management" illustrates many important applications of HFSWR in improving the management of the coastal environment such as pollution control and tsunami monitoring.

Although only one paper associated with microwave radar research is collected in this special issue, the interest in such radar has been increasing significantly. In the paper titled "Study of Ocean Waves Measured by Collocated $\mathrm{HH}$ and VV Polarized X-Band Marine Radars," significant wave height estimates from marine radars with different polarization have been compared against buoy measurements. The results obtained with VV polarization are found to be more accurate, especially under low wind conditions.

\title{
Acknowledgments
}

The editors would like to thank all the authors for contributing their interesting work associated with the topic of this special issue and the anonymous reviewers for their time and effort expended on providing valuable comments.

\author{
Weimin Huang \\ Xiongbin $W u$ \\ Björn Lund \\ Khalid El-Darymli
}




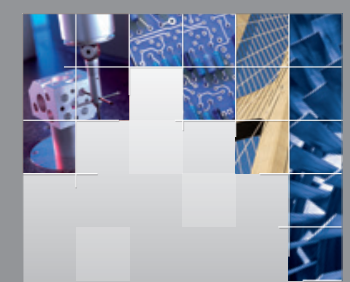

\section{Enfincering}
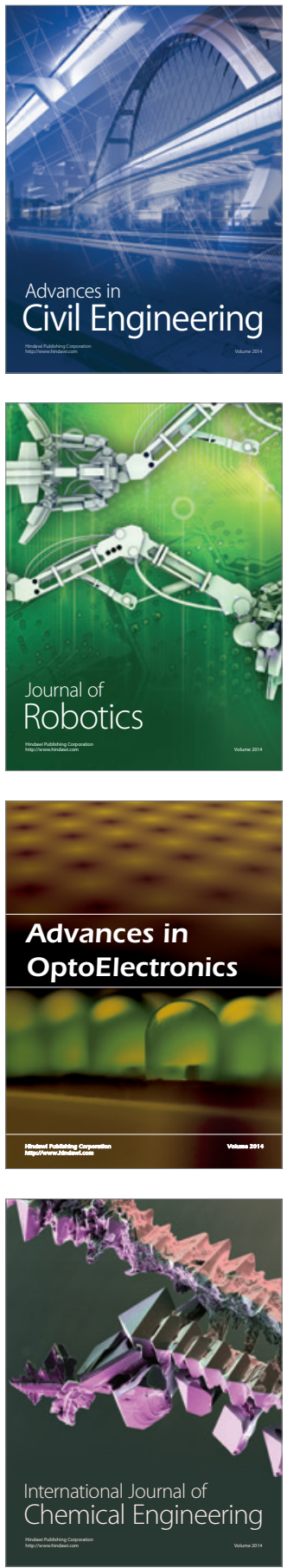

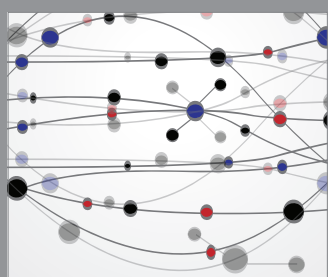

The Scientific World Journal

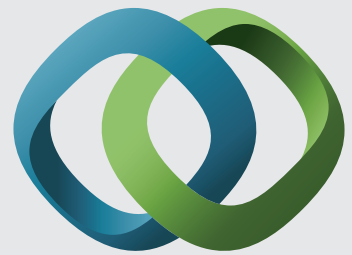

\section{Hindawi}

Submit your manuscripts at

https://www.hindawi.com
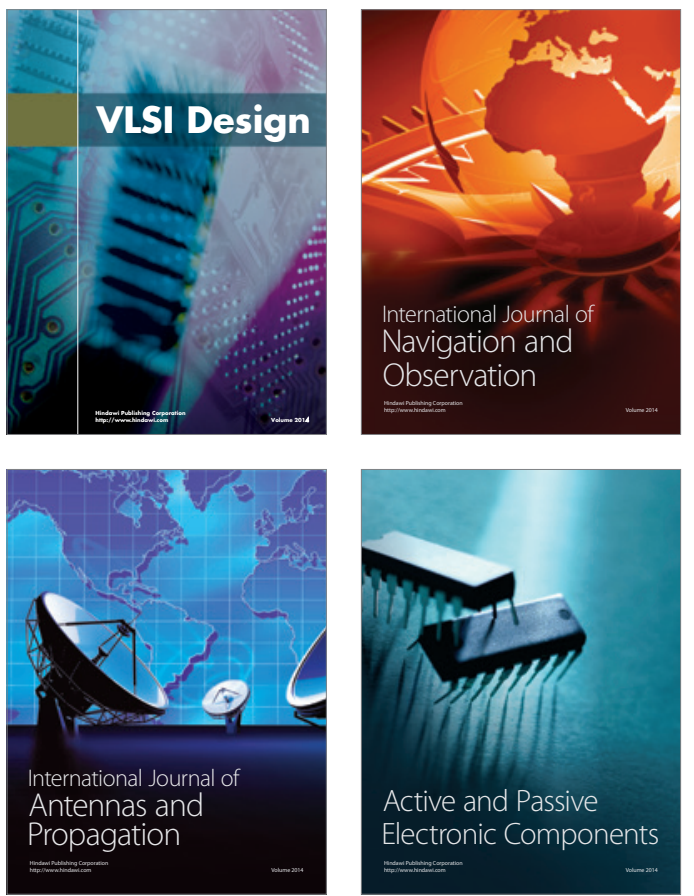
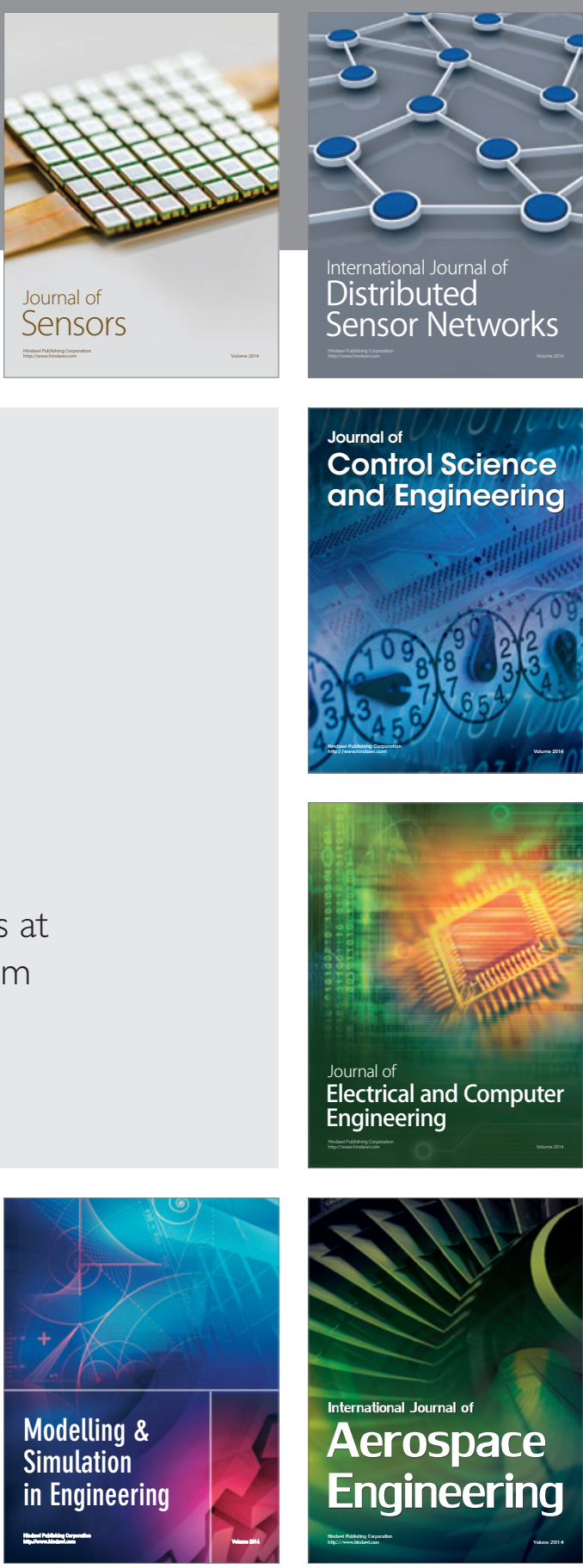

International Journal of

Distributed

Sensor Networks

$-$

Joumal of

Control Science

and Engineering
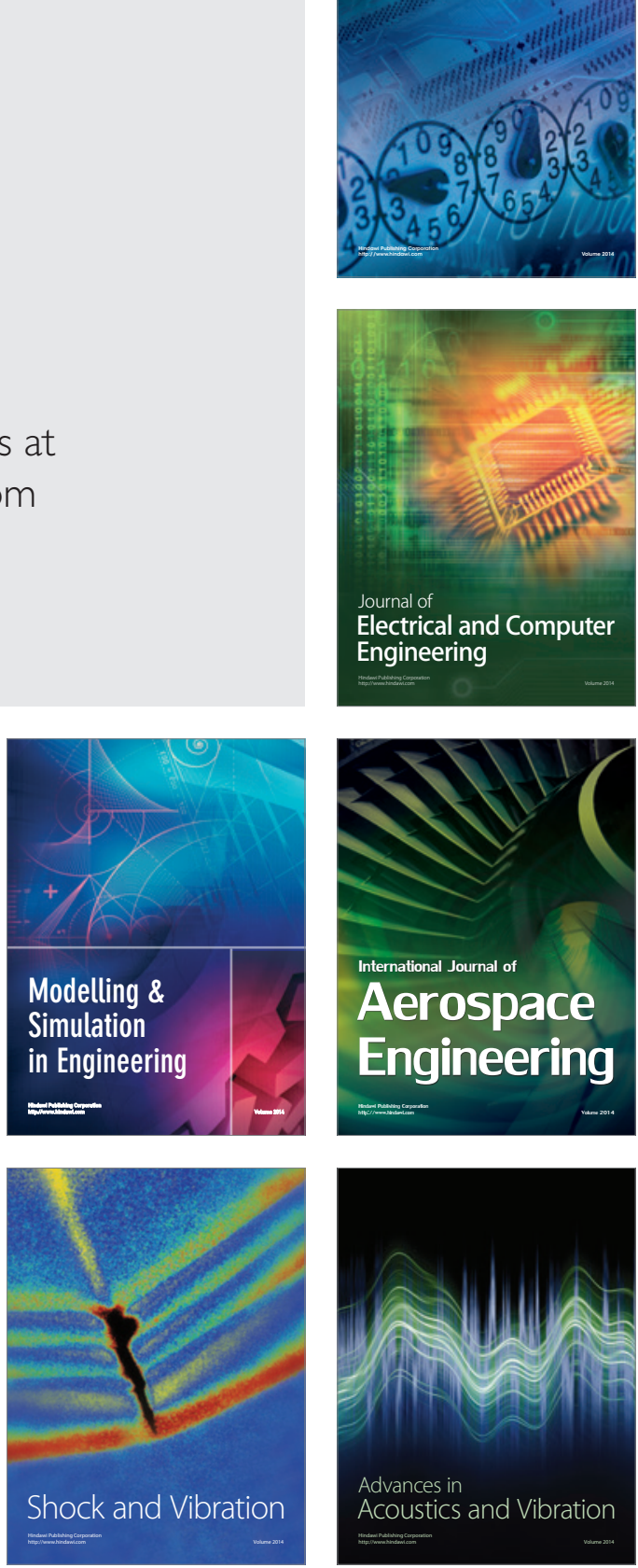